\title{
Research on the Interactive Intelligent Hardware System Based on UI and UE Enhanced Design Pattern
}

\author{
Zuliang Wang*, Chao Zhang, Zhangyun Jin, Xiaoying Liu, Zhipeng Chen, \\ Tiantian Zhang, Liangliang Ma and Hao Wang \\ Department of Electronic and Information Engineering, Xijing University \\ 1601252678@qq.com
}

Keywords: Interactive; UI and UE; Design pattern; Hardware system; Design and implementation

\begin{abstract}
In this paper, we conduct research on the interactive intelligent hardware system based on UI and UE enhanced design pattern. Man-machine coordinated interaction environment, reasonable division of labor is people thinking activities as much as possible that are the basic main task of the computer of the information filtering, processing, and in the form of easy decision maker use. Many research institutions and researchers are working to develop suitable devices for virtual reality and teleoperation system efficient human-computer interface device. Our research combines the latest and state-of-the-art perspectives to propose the related new method that is helpful.
\end{abstract}

\section{Introduction}

Smart hardware industry in the early stage of the product definition, application field prepared after segmentation, capital injection, industry chain that is entering a rapid development stage. Information technology enterprise and the electric appliance, automotive, medical and health care industries such as cooperation, through the key node of the hardware product development and the application of the bottleneck. Intelligent dressing equipment, smart home, intelligent car as intelligent robots and other hardware products and application development will presents the fast growth.

The reliability of the intelligent instrument is decided by two aspects of hardware reliability and the basic software reliability, in general, the software reliability of intelligent instrument is higher, so the reliability of the intelligent instrument is mainly determined by hardware reliability. (1) The reliability of the components. Intelligent instrument is often composed of the multiple circuit boards and each circuit board is composed of multiple components, therefore, intelligent instrument faults are caused by components damaged or the variable value more based on all kinds of the component under certain conditions a large amount of experimental analysis shows that components of fault have the certain regularity. (2) Adopted by circuit structure factors while the more components, the reliability of the intelligent instrument is the worse. For intelligent instruments, adopts for the integration of the large integrated circuit can reduce the number of components and improve the reliability of basic intelligent instrument. (3) Components manufacturing process factors. In the process of intelligent instrument production, the more automated processes, the higher the degree of automation, its fault is less as the higher the reliability of the intelligent instrument [1-3].

Whether one can be a very good interactive user operation mood and perception depends largely on the stand or fall of the man-machine interaction software interface design. Design train of thought and direction directly affects the use of software as takes root in the user's heart will have unforgettable. In analysis more accurate, design quality and even affect the rest of the hardware such as performance, is related to the structure of the whole software integration system level, it is very important.

Information visualization in for a given type of data to create the right view and effective interactive technology, is the use of abstract data representation of the computer support, interaction, visual, its research purpose lies not in the graph itself, but is to strengthen the cognitive and the insight. Easy to policymakers' cognitive information form of reasonable can help people quickly find information and digest information quickly and effectively monitor the huge amounts of information. 


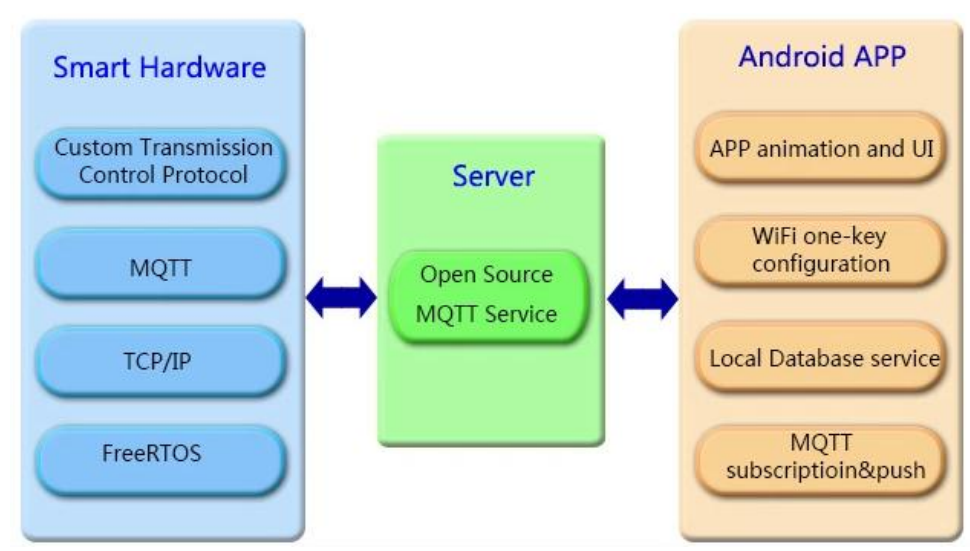

Figure 1. The Traditional Architecture of the Intelligent Hardware System

In this paper, we conduct research on the interactive intelligent hardware system based on UI and UE enhanced design pattern. Man-machine coordinated interaction environment, reasonable division of labor is people thinking activities as much as possible that are the main task of the computer of the information filtering, processing, and in the form of easy decision maker use. According to the people in the process of the information presentation thinking characteristics of decision-making activities to provide policy makers natural interaction and helps decision-makers information expediently.

\section{The Proposed Methodology}

The User Experience Design. User experience is new interdisciplinary, and grows with development of design, study, psychology and sociology, design engineering and other academic disciplines have inalienable relations. It is the user in the process of using product built a purely subjective feeling as including all kinds of complex psychological feelings [4].

User experience occurred in the process of the interaction, interactive object is the user, product or system, and cover scope includes product or system availability, appearance design, user emotion, motivation, emotion that various kinds of the factors that affect the user experience. User experience design based on user research as the center, from the perspective of the product users, embodies the concept is a process. From this point of view, the object of the user experience design is not only a product or service itself, but rather the product or service of the entire process of user experience, runs through the whole life cycle of a product or service.

The era of experience economy the user experience is not only to meet the needs of basic users to function, at the same time, also need to satisfy user's physiological and psychological needs, bring joy full of personalized experience to the user. User experience research purpose is to affect the user experience design factor, and general optimization of product, service, or some corresponding system, in order to improving the quality of the user experience, and enhance customer loyalty. To see these things or concepts as one by one point, when a point is activated and the other is linked to the point quickly activated. Only when the relationship between point and its activation in accordance with the user's cognitive structure and emotional needs, the user will be able to understand things or concepts, and get a positive user experience that could be summarized as the following aspects.

- The style of the interface need to overall consideration, first of all, according to the interface integral style planning ideas, and combining with the need of interface on other elements, to ensure the convergence of icons and the overall effect.

- Icon design is based on function to mark. The purpose is to make practical and basic beautiful design logo. Icon of the icon should consider the practicality of basic metaphorical, it convey the meaning must be the user, known as unknown.

- For high fidelity color reduction degree has certain limitations, so when choosing color have to be constantly adjust according to the use of the screen better approach is to use mobile phones take pictures of the browser to full screen view high fidelity. 
This series of solid foundation should be solid user research. User experience design provides the user the new field of vision provide theory support for the innovation of product design and practical foundation only starting from the source culture structure of general user experience, user experience research model, user model, design model and a virtuous cycle between products that can improve the level of theory and practice of industrial design [5].

The Human-Computer Interaction. Design an interactive system that must be based on the clear guidelines for the design of a complete and thorough and detailed. This interface system designed with the object-oriented method, so that the interface structure clear, that easy to operate, and has the characteristics of good scalability, and its design principle and method human-computer interaction interface is versatile to build larger systems.

From the perspective of ergonomics, the user use the software is more difficult to solve, so in the process of evaluation of interacting prone to a common mistake. With the professional can grasp the degree of words, for example as make the expression is relatively professional and easy for users to identify. When operated in different users will encounter all sorts of problems, which requires for the solution of problem has a very rigorous analysis system. Many research institutions and researchers are working to develop suitable for virtual reality and teleoperation system efficient human-computer interface device and try to take various methods to force the sensing reappearance function is added to the system, in the face of different kinds of force sensing device to be introduced.

- Data glove and wear equipment. Wearable devices than exoskeleton much more freedom of movement that is the miniaturization of exoskeleton, mostly in the form of data glove. Not as exoskeletons and stationary equipment restricted by the force of gravity, its biggest advantage is that can be in a natural way for human-computer interaction which can strengthen people in immersive virtual environment.

- Force sensing devices based on intelligent materials. Force sensing reproduction device can actually as a "stretch" of the computer, can impose physical force and moment on the operator, comparison of advantages and disadvantages for methods of implementation reproduction.

- Exoskeletons and capital equipment. There is a lot of exoskeleton interactive equipment can provide the whole arm or other parts of the body force feedback information, including Iowa force feedback information provides the user with a force of the magnetic fields exoskeleton.

The Intelligent Hardware System. Intelligent product architecture as a whole, if the abstract into several levels, at present, the products mainly in the bottom layer as the part is divided into hardware and software control processor and network interconnection layer, is among the products used for networking, when air conditioning intelligent WIFI access module to realize the function of basic networking and the top is the real "brain", namely intelligent clouds, all kinds of basic services and applications in the cloud, with the aid of the data analysis and dig with intelligence services.

As mentioned earlier, the current products are implementing networked control, but did not reach the level of connectivity, and the artificial intelligence, so all sorts of products are chasing intelligent hardware trend. In all of the interconnected age, intelligent hardware products can achieve natural interaction, independent study, connectivity, etc. Man and the machine interaction will get rid of the current depends on the current situation of the mobile phone App, toward the voice, gestures and the other natural interaction of a variety of basic ways. People can in the most natural state, with the most natural way to achieve communication with intelligent hardware products [6].

Part of intelligent hardware business at first, the product positioning is not accurate enough, after a period of groping, repositioning of the product, and then find a suitable location and smart hardware development needs analysis could be summarized as the follows. (1) Collaborative development becomes the inner needs of the development of the intelligent hardware innovation. Smart hardware is not only a hardware device with the need to interact through data interaction and the cloud to achieve powerful features, innovation is hardware and software integration, and infiltration to many industry fields. (2) Smart hardware lack of uniform standards, the product service innovation quality is uneven. At present, different brands of smart hardware equipment collected data format and the data quality differences, lack of data sharing and integration. Through data sharing and interdisciplinary 
integration, create new applications and services to promote the flow of information and sharing. (3) At present, the intelligent hardware participants mainly small and basic medium-sized enterprises and individual developers, more research and development spending less, lack of environment. In light of the present condition of the open source software and hardware tools resource diversity, because there is no systematic combing with popularity and effective dissemination [7].

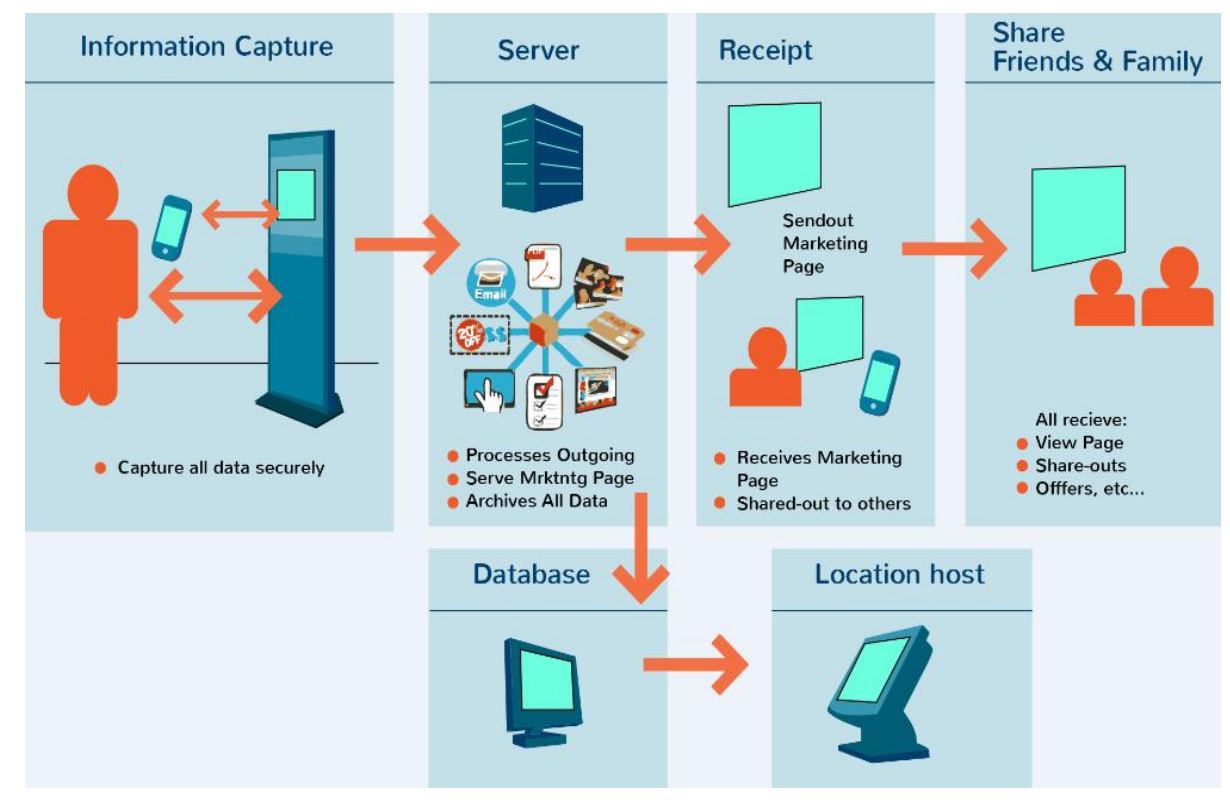

Figure 2. The Interactive Intelligent Hardware Pattern

The Software Interface Optimization. Interface is mainly made up of the menu, the menu has wide application in interactive software such as generation, many system software not only provide a system menu operation, but also provide some menu generation tool, allowing users according to their own need to generate their own application level control system to realize link between father and son menu, implement a menu item with the corresponding link between an executable program and phase separation is the realization of the software to open the menu and process, the path of evolution.

Different types of user interface of the software system forms have different habits and the basic requirements. In general, the user can be divided into professional users and ordinary users. As for the mentioend interfaces, we should follows the listed suggestions for better optimization. (1) Functional interface. For functional interface, it is property content, for any software product and the value of its existence is the practicability of the first. People exchange information through the software interface and software to use the software function, instead of software function through software interface. (2) Situational interface. Any design will be linked to environmental factors, it includes integrated areas such as social, political, and cultural. Environmental factors of basic interface design covers is very extensive, it include the politics, history, economy, culture, science and general technology, national environment design of the interface are embodies the sociality of design art. (3) Affective interface. The development of modern semiotics is increasingly to this field, in an general effort to make this uncertainty compression, and strengthen the rational composition. Semiotics is widely used in various fields, such as the symbol, the research of the semiology to epistemology investigation understanding perception, semiotic problems in the process of cognition [8].

Interface effect is the embodiment of human-computer interaction interface effect. Text and black and white it's easy to cause the users quickly fatigue such as color, image media interface can increase the visual appeal, reduce fatigue; Graphics is more intuitive, image, the advantages of large amount of information. Therefore, the use of multimedia to show some entities or operation, metaphor, can the user's operation and its look and feel more immediate, visible, and clear, enhance understandability of the software system and easy to use. Human-computer interaction feedback refers to the user in the process of man-machine operation users get information from the software system. Feedback reflects 
the software response to user interaction action done, to allow the user to determine the effect after operation. So that design human-computer interaction interface must be considered system feedback information to the general user action.

\section{Conclusion}

In this paper, we conduct research on the interactive intelligent hardware system based on UI and UE enhanced design pattern. Human-computer interaction technology is becoming a hot spot of terminal brands competition, voice control, basic face recognition, gesture, augmented reality, eye-tracking new human-computer interaction technology constantly emerging, including the application of voice control technology is relatively extensive several other kinds have become new application direction. In is paper, we review the latest UI and UE enhanced design pattern and apply it into the interactive intelligent hardware system design and implementation that is innovative. In the future research, we will enhance the current methodology with the theoretical optimization.

\section{References}

[1] Tuntas, Remzi. "A new intelligent hardware implementation based on field programmable gate array for chaotic systems." Applied Soft Computing 35 (2015): 237-246.

[2] Popov, Serge, et al. "Hardware and software equipment for modeling of telematics components in intelligent transportation systems." Internet of Things, Smart Spaces, and Next Generation Networks and Systems. Springer International Publishing, 2014. 598-608.

[3] Chen, Pei-Yin, et al. "An efficient hardware implementation of HOG feature extraction for human detection." Intelligent Transportation Systems, IEEE Transactions on 15.2 (2014): 656-662.

[4] Sankaran, R., et al. "Waggle: A Framework for Intelligent Attentive Sensing and Actuation." AGU Fall Meeting Abstracts. Vol. 1. 2014.

[5] Cintuglu, Mehmet H., Harold Martin, and Osama A. Mohammed. "An intelligent multi agent framework for active distribution networks based on IEC 61850 and FIPA standards." Intelligent System Application to Power Systems (ISAP), 2015 18th International Conference on. IEEE, 2015 .

[6] Stephen, Valantina, and L. Padma Suresh. "FPGA-based Intelligent Control of AC Motors." Artificial Intelligence and Evolutionary Algorithms in Engineering Systems. Springer India, 2015. 459-471.

[7] Mzahm, Anas M., Mohd Sharifuddin Ahmad, and Alicia YC Tang. "Agents of Things (AoT): An intelligent operational concept of the Internet of Things (IoT)." Intelligent Systems Design and Applications (ISDA), 2013 13th International Conference on. IEEE, 2013.

[8] Zeigler, Bernard P. Object-oriented simulation with hierarchical, modular models: intelligent agents and endomorphic systems. Academic press, 2014.

[9] Wang, YuLing, Ming Li, and Li Li. "An image denoising algorithm based on clustering and median filtering." Sixth International Conference on Graphic and Image Processing (ICGIP 2014). International Society for Optics and Photonics, 2015.

[10] Shettar, Jyoti, Ekta Maini, and S. Shreelakshmi. "Image Sharpening \& De-Noising Using An Adaptive Bilateral Filter." International Journal of Innovative Research and Development 2.11 (2013). 